\title{
SEAloT: Scalable E-Health Architecture based on Internet of Things
}

\author{
Omar Said \\ Department of Information Technology, Taif \\ University, Taif, 888, Saudi Arabia
}

\author{
Amr Tolba \\ Department of Computer Science, King Saud \\ University, Riyadh, 11437, Saudi Arabia
}

\begin{abstract}
In this paper, a scalable e-health architecture based on the Internet of Things (IoT) technology is proposed. In this suggested architecture, the clinic hardware can communicate with other clinics' hardware remotely with minimal or no human intervention, which is a key difference between the proposed architecture and the former ones. The idea of our architecture is to use the internet as a communication media between different architecture hardware. Each hardware clinic should be adapted to receive its required information from the internet. Using our system will provide the medical specialists with advantages such as scalability and flexibility. Furthermore, the cost and the effort will be decreased. The processes in our proposed architecture are executed on advanced medical devices in real time mode, which maximize the diagnosis accuracy and minimize the processing time. Finally, a case study, which is related to $2 \mathrm{D}$ and 4D ultrasonography devices, is demonstrated to clarify our architecture.
\end{abstract}

\section{Keywords}

E-Health, Internet of Things, Intelligent Web Application, Cloud Computing.

\section{INTRODUCTION}

In spite of good intention, health systems are unable to deliver high-quality with inexpensive services to humans. Huge numbers of people die each day without being able to avoid a disease in the first place. Quality of care is inferior. Patients may get to a clinic, but they face long waits. Thick medical files cause the most important and critical health data to be buried. In addition, services are overwhelmed with extremely severe shortages beyond repair. One of the greatest challenges which face the general public is the inequities in terms of health care. This demand can be satisfied in the near future. Hence the e-health field is introduced to study this challenge and solve its problems $[1,2]$.

There is a wide range of implements in the e-health world, such as software for data gathering, mobiles, etc. Combining these things in one system may be slightly health disparities, which enable massive leaps in quality of care. For example, using a laptop or a mobile device, which became mostly available, anybody can access the best treatment information and can keep track of their medical case(s) using electronic health records. However, this facility was available to only rich people. There are some queries, which are raised in ehealth care systems. Among these queries, we find: does applying these technologies actually improve the patients' health? And does e-health really has a collision on the patients' health status?
Recently, the concept of the Internet as a set of connected computer devices is changed to a set of connected surrounding things of human living space, such as home appliances, machines, transportation, business storage, goods, etc. The number of things in the living space is larger than the number of the world population. Research is heading toward finding out how to make these things to communicate with each other like computer devices communicate through the Internet. The communication among these things is referred to as the Internet of Things (IoT). In this paper, we apply IoT idea on the heath care systems to create a new e-health generation $[3,4,5]$.

This paper proceeds as follows; in Section 2, the related work evaluation is introduced. In Section 3, our proposed architecture is demonstrated. In Section 4, a case study that contains a communication between 2D and 4D ultrasonography devices is shown. In Section 5, the expected advantages, which may be extracted from our proposed architecture implementation, are determined. Finally, the conclusion and future work will be presented in Sections 6 and 7 respectively.

\section{RELATED WORK}

In this section, we briefly present some of the research that is related to our work. Monika Grajzer et al., [2] presented one of the recent machine learning-based techniques for identifying and detecting traffic on mobile e-health applications. These mobile e-health applications can be improved by providing a multi-classification technique to determine traffic generated by its users. The researchers on [6] found that this solution can be applied to other applications that are not related to e-health. Furthermore, time, complexity, and efficiency are not measured.

A Multi-Agent System for small e-Health care organization has been introduced by [7]. This paper uses JADE as an agent-oriented framework for building e-Health system, and points out that the adding of agent features allow the e-health system to be more intelligent, flexible, and active. In our point of view, these features may add some complexity to the system. On the other hand, the author of [8] embedded the concept of cloud computing in e-Health and suggested a cloud-based e-health framework that uses multimedia as video coding for heterogeneous network. In this framework, the transmission of health care services and monitoring resources behaviors are based on cloud computing concepts. This article doesn't mention the complexity and network overhead factors in the simulation result. A PAAS framework of privacypreserving authentication system in e-health network has been presented by [9]. The authentication strategies with privacy requirements between patients are also offered by PAAS. The 
researchers of [9] compared their framework with existing ehealth systems only in terms of privacy and ignoring cost and communication overhead factors. In the same way, the authors of [10] proposed a security and privacy scheme that uses different techniques to protect patients' data against attack in the e-health systems.

Yang Guo et al., [11] introduced the concept of interoperability and its use of e-health applications. A case study in Blekinge County Sweden healthcare organizations has been analyzed to understand the concept of interoperability. Also [11] introduced a peer to peer model to solve the problem of interoperability on e-health systems. This trial focuses on syntactic interoperability and discards semantic interoperability due to the need of high level of security and privacy. A comprehensive overview of existing wireless techniques that are used in e-health systems has been introduced by [12]. This review article provides some techniques that can be used in e-health systems toward an interoperability framework; it gives us a good starting point to understand this concept. The closed related work, which discussed by [13], discuss the overloading data which may be caused by IoT system. Hence, the e-health system is taken as an example.

Based on the above related works, we can realize that most of the preceding models and frameworks are implicitly structured and lacks some components to control the constituted e-health system.

\section{OUR PROPOSED ARCHITECTURE}

Our proposed architecture deals with the medical devices which operate by the information feeding and may not require the patient's body. The architecture contains three main components; the internet as a communication media, the Radio Frequency IDentification (RFID) that will be used to read and distribute the environment information [4], the hardware which should be adapted and used in our e-health architecture, and an intelligent web application.

The Internet is a famous communication media that is available all over the world. So, the distance between the clinic hardware is not a restriction. The RFID will be used to collect the data which is required for machine-to-machine communication process. The RFID is attached to the system hardware. The architecture hardware will be cooperated in harmony to raise the proposed e-heath system efficiency. The intelligence web application will be used to manage the entire system. In our system, some components are well-defined and didn't need more descriptions such as the Internet. The other components; RFID, Hardware, and a web application should be briefly described.
RFID is used to transmit the collected data using a portable device called a tag. The transmitted data can be identified by the real location of hardware. The RFID is not a new technology as it is used by thousands of companies. RFID advantage is used to track the state of each object in the architecture. Another advantage to it is its ability to retrieve the data stored on an RFID tag, which is a reader device that has one or more antennas and emits radio waves, will be used. Then, the reader passes the information form to a computer system. The hardware which will be used in the e-health system should have a crucial condition. This condition is that the job or target of the two hardware components, which will be communicated using IoT technology, should have harmony or compatibility in order to accomplish this target. Alternatively, the architecture contains one hardware component that is divided into two parts, which should be ready for communication over a wide space. In addition, how the IoT hardware will send and retrieve the information from the RFID devices (tag and reader) should be studied.

The intelligent web application can be considered as the core of our proposed architecture since it is used to manage other components. This application should comprise the software catalog of each architecture device. This catalog describes how these components are working and communicating with each other. The application should be intelligent to eliminate the human intervention while the system works. Furthermore, this application should be aware of regular or sudden major or minor system changes. In addition, it should be standardized and platform independent. No human intervention, which is a general target for most IoT systems, is considered an important advantage as it makes the architecture processes done automatically with high sensitivity as a result of calling the high-efficiency medical devices remotely.

The core idea of this architecture is that the hardware sends the information which is gathered by RFID using a wireless Internet connection. Hence, other hardware components will receive this data, which is required in processing. The data is gathered directly from patients or remotely by other RFID hardware. Upon this strategy, we can detect that the input of each health care device is the keystone of our idea. Since each hardware device in the old e-health system was worked alone and must require the patient, the main advantage, which can be briefly stated "the specialists can easily use modern and advanced medical devices, which are found throughout the world," is eliminated. For our proposed architecture hardware distribution and its simple view, see Fig1. To clarify the core difference between the old health architecture and our proposed one, see Fig. 2 and Fig 3. 


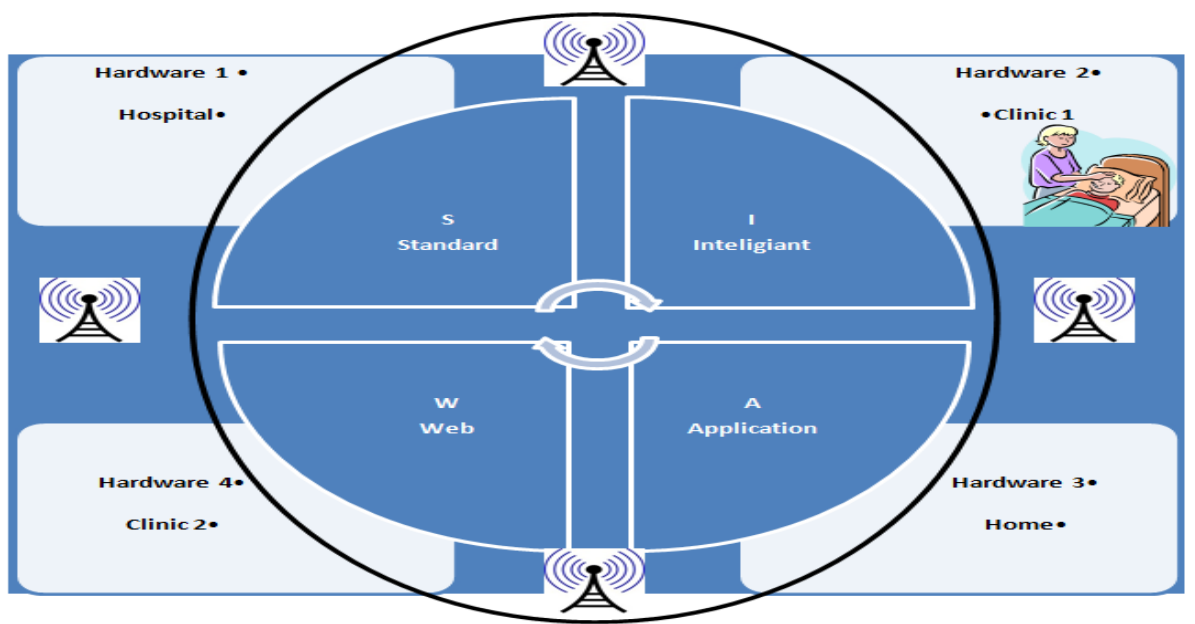

Fig 1. General view of our proposed architecture.

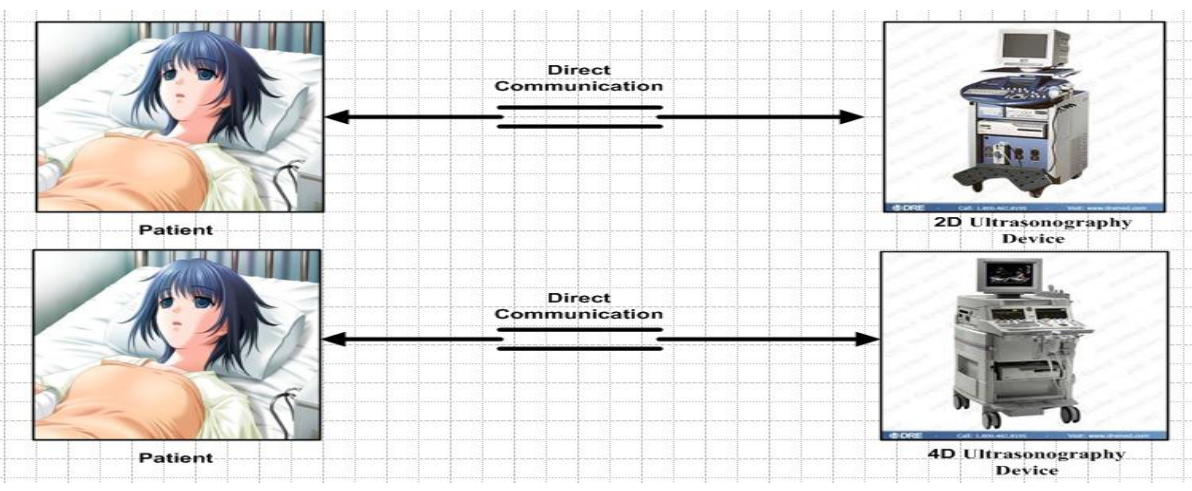

Fig 2. General view of old architecture.



Fig 3. General view of our architecture.

\section{CASE STUDY (2D AND 4D \\ ULTRASONOGRAPHY DEVICES)}

Our case study includes three outpatient clinics in three different places. The hardware components, which will reflect the main target of our system, are 2D and 4D ultrasonography devices [14], [15]. The 2D ultrasonography device will be placed at outpatient clinic $X$. The 4D ultrasonography device will be placed at outpatient clinic Y. The medical specialist will be present at outpatient clinic $Z$. There is no restriction on the distance between the three outpatient clinics. In the current study, four communication states should be identified.

The first communication state occurs between the patient and the 2D ultrasonography device. The patient should lie behind the 2D ultrasonography or the specialist can remotely control the $2 \mathrm{D}$ probe part. Hence, the patient may select two types of communications; direct or indirect. In the direct one, the patient may attend by himself to the 2D ultrasonography device and it is recommended to the not bad patients' health state. In the indirect one, the patient may use the probe of the $2 \mathrm{D}$ 
ultrasonography device and the extracted data can be transferred by the internet (till now this state is not real).

The first communication state occurs between the patient and the 2D ultrasonography device. The patient should lie behind the $2 \mathrm{D}$ ultrasonography or the specialist can remotely control the $2 \mathrm{D}$ probe part. Hence, the patient may select two types of communications; direct or indirect. In the direct one, the patient may attend by himself to the $2 \mathrm{D}$ ultrasonography device and it is recommended to the not bad patients' health state. In the indirect one, the patient may use the probe of the 2D ultrasonography device and the extracted data can be transferred by the internet (till now this state is not real).

The second communication state occurs between the patient and the specialist. In this situation, a web digital camera should be attached to transfer real time video signals to the specialist using a multimedia application which may consider as a part of the general web intelligent application. The third communication state should be done between the $2 \mathrm{D}$ and the 4D ultrasonography devices. This is considered the most important state due to sensitivity in data processing, extraction, transmission, and retrieving by the specialist in $4 \mathrm{D}$ styles. This state can be discussed as follows; the 2D starts to extract the video data from patient. Accordingly, the extracted data are handled and stored in a buffer. Hence, the management web application will use a multimedia communication protocol such as RTP [4], [16] to send these data through the internet.

The fourth communication state occurs between the specialist and the 4D ultrasonography device. This state should be done remotely. The results, which should be acquired from the third communication, should be transferred to the specialist in real time mode. Upon these results, the specialist can start to diagnose the patient. The 4D ultrasonography device will receive the discrete video sectors, which were extracted from the patient. Upon the idea of 4D ultrasonography device, the video sectors can be combined into one $4 \mathrm{D}$ video. Hence, the specialist can read and analyze these data and get accurate diagnose.
It's notable that there are many obstacles which may be faced if we implement this case study. These obstacles can be stated as follows; 1) The 2D ultrasonography device should be adapted to contain a wireless system between the probe and its central processing unit. Furthermore, it should contain a buffer to store the extracted patient information. In addition, this device should act as a wireless node which can send and receive control and diagnostic data. 2) The patient should have lower limit of experience in dealing with our system especially if he wants to gain the maximum advantages from our architecture. 3) The 4D ultrasonography device should be adapted to receive the data which will be sent from the 2D ultrasonography device and also it must work as a wireless node [17].

These obstacles will be solved by the remarkable and rapidly developing technology in medical devices. For the scenarios description between the proposed architecture components, see table 1 . The general view of our case study is found in Fig. 4

Table 1. Communication states description of our proposed architecture components

\begin{tabular}{|c|c|}
\hline Scenario & Complexity \\
\hline $\begin{array}{c}\text { The patient settled behind the 2D } \\
\text { ultrasonography device }\end{array}$ & Simple (Realistic) \\
\hline $\begin{array}{c}\text { The patient settled at home and can use } \\
\text { the wireless probe. }\end{array}$ & $\begin{array}{c}\text { Simple (Waiting } \\
\text { for technology) }\end{array}$ \\
\hline $\begin{array}{c}\text { The specialist settled behind the 4D } \\
\text { ultrasonography device with state 1. }\end{array}$ & Simple (Realistic) \\
\hline $\begin{array}{c}\text { The specialist settled behind the 4D } \\
\text { ultrasonography device with state 2. }\end{array}$ & $\begin{array}{c}\text { Simple (Waiting } \\
\text { for technology) }\end{array}$ \\
\hline $\begin{array}{c}\text { The specialist settled behind the 2D } \\
\text { ultrasonography device with state 1. }\end{array}$ & Simple (Realistic) \\
\hline $\begin{array}{c}\text { The specialist settled behind the 2D } \\
\text { with state 2. }\end{array}$ & $\begin{array}{c}\text { Simple (Waiting } \\
\text { for technology) }\end{array}$ \\
\hline $\begin{array}{c}\text { The specialist settled far away from the } \\
\text { 2D with state 1. }\end{array}$ & $\begin{array}{c}\text { Difficult (Waiting } \\
\text { for technology). }\end{array}$ \\
\hline $\begin{array}{c}\text { The specialist settled far away from the } \\
\text { 2D with state 2. }\end{array}$ & $\begin{array}{c}\text { Extremely } \\
\text { difficult (Waiting } \\
\text { for technology). }\end{array}$ \\
\hline
\end{tabular}

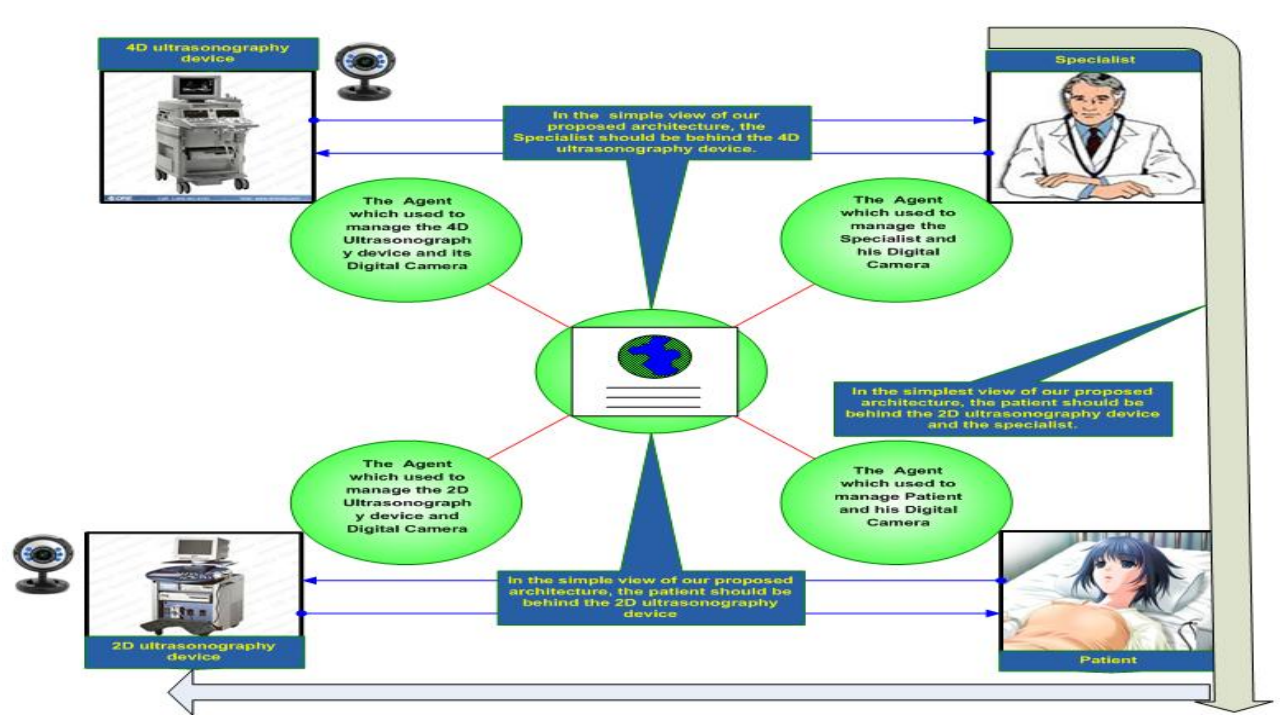

Fig 4. General view of our case study. 


\section{EXPECTED ADVANTAGES}

1. The cost of clinics is decreased because the system may use a central medical device which can serve outpatient clinics or hospitals.

2. The e-health system efficiency is increased because most of medical specialists can use advanced devices remotely which will enhance the ability to reach the proper diagnosis.

3. No (or minimal) human intervention.

4. The distance between the clinics is not a restriction.

5. The proposed architecture is scalable because we can extend the architecture to include more devices in different places.

6. Internet, which is used as communication media, is available approximately to everybody in different forms (Mobile, Home, Work, Business, etc.).

\section{CONCLUSION}

In this paper, we introduced an e-health architecture based on the IoT technology. In this architecture, the medical specialist can use and control advanced medical devices remotely using machine to machine communication. In addition, the medical specialist can watch the patient status using an intelligent IoT room with a digital camera. Furthermore, the patient may use our architecture by attending to the clinic or can access remotely from his position. A case study, which describes how 2D and 4D ultrasonography devices are communicated regardless the distance between them, is demonstrated to clarify our proposed architecture. Some obstacles that may be faced during implementation of our case study are introduced. Finally, the expected advantages of our proposed architecture are determined.

\section{FUTURE WORK}

The next step of our work contains two main procedures. The first procedure is to define a protocol which clarifies how the machines will be remotely communicated and how the system will be worked. The second procedure is to prove that our architecture is applicable. This can be accomplished by implementing the suggested case study. Hence, the architecture components should be precisely determined and analysed. Upon this implementation, many of the above stated obstacles may be faced and tolerated by decreasing the expected advantages from the patient side.

\section{REFERENCES}

[1] Royal College of Nursing, eHealth survey 2010 report.

URL:http://www.rcn.org.uk/_data/assets/pdf_file/0005/3 91109/004115.pdf

[2] Monika Gajzer. et al, A Multi-Classification Approach for the detection and Identification of eHealth Application. IEEE International Conference on Distributed Computing Systems (ICDCS), 18 - 21 June 2012

[3] Yuxi Liu, Guohui Zhou, Key Technologies and Applications of Internet of Things, IEEE Fifth International Conference on Intelligent Computation
Technology and Automation, Hunan China, pp: 197-200, 2012.

[4] Luigi A., Antonio I., Giacomo M., The Internet of Things: A survey. Science Direct journal of Computer Networks, Volume 54, Pages: 2787-2805, 2010.

[5] Yinghui H., Guanyu L., Descriptive Models for Internet of Things. IEEE International Conference on Intelligent Control and Information Processing, Dalian, China, Pages: 483- 486, 2010.

[6] Monika Gajzer. et al, A Multi-Classification Approach for the detection and Identification of eHealth Application. IEEE International Conference on Distributed Computing Systems (ICDCS), 18 - 21 June 2012.

[7] R. Jaya Subalakshmi. et al, A Small e-Health Care Information System with Agent Technology. IEEE International Conference on Computational Intelligence and Communication Systems, 7-9 Oct. 2011.

[8] Atif Alamri, Cloud-Based E-Health Multimedia Framework for Heterogeneous Network. IEEE International Conference on Multimedia and Expo Workshops, 9-13July 2012.

[9] Linke Guo. et al, PAAS: A Privacy- Preserving Attributebased Authentication System for eHealth Network. IEEE International Conference in Computer Communication and Networks (ICCCN), July 30 - August 2012.

[10] Papalambrou, A. et al, Communication security and privacy in pervasive user-centric e-health systems using Digital Rights Management and side channel attacks defense mechanisms. IEEE International Conference on Industrial Technology (ICIT), 19-21 March 2012.

[11] Yang Guo. et al, Using P2P Technology to Achieve eHealth Interoperability. $8^{\text {th }}$ IEEE International Conference on Service Systems and Service Management (ICSSSM), 25 - 27 June 2011.

[12] Antonio Aragues. et al, Trends and Challenges of the Emerging Technologies toward interoperability and standardization in e-Health communications. IEEE Communications Magazine, Vol 49 Issue: 11 ISSN: $0163-$ 68042011

[13] Martin Diaz, et al., Big Data on the Internet of Things An example for the E-health. Sixth International Conference on Innovative Mobile and Internet Services in Ubiquitous Computing, pp: 898-900, Palermo, Italy, 4 to 6 July, 2012.

[14] Tim Irwin, Luong Banh, Software Visualization of 4D Ultrasound Data.

URL:http://www.timirwin.com/Projects/4D\%20Ultrasoun d\%20Visulazation.pdf

[15] P Soma-Pillay, H Lombaard, 2D, 3D, 4D ultrasound in Obstetrics: Best evidence practice. Obstetrics \& Gynaecology Forum, Vol 20, No 2, pp: 56-58, 2010.

URL:http://137.215.9.22/bitstream/handle/2263/14215/So maPillay_2D(2010).pdf?sequence $=1$

[16] Liang Z., Han-Chieh Chao, Multimedia Traffic Security Architecture for the Internet of Things, IEEE Networks, 2011. Vol.25, No. 3, Pages: 35-40, 2011.

[17] Vasseur J., Dunkels A., Chapter 4 - IPv6 for Smart Object Networks and the Internet of Things, Elsevier Interconnecting Smart Objects with IP, Pages: Pages 3949, 2010. 